\section{THU0274 RENAL CHARACTERISTICS AND OUTCOME OF LUPUS NEPHRITIS ACCORDING TO ITS TIME OF ONSET}

O. C. Kwon ${ }^{1}$, J. H. Park', M. C. Park'. ${ }^{1}$ Yonsei University College of Medicine, Division of Rheumatology, Department of Internal Medicine, Seoul, Korea, Rep. of (South Korea)

Background: Lupus nephritis (LN) usually develops within 5 years of systemic lupus erythematosus (SLE) onset. It is unclear whether the course and outcome of LN differ between patients who initially had LN at SLE onset (initial-onset LN) and those who developed LN within 5 years after SLE onset (early-onset LN).

Objectives: To compare clinical characteristics and renal outcomes between SLE patients with initial-onset LN and SLE patients with early-onset LN.

Methods: SLE patients with biopsy-proven LN were retrospectively reviewed. The clinical parameters and renal outcomes were compared between initial-onset $\mathrm{LN}$ and early-onset $\mathrm{LN}$ groups. We used Cox regression analysis to estimate risk of worse renal outcome, according to the onset time of LN. Results: Of the total $136 \mathrm{LN}$ patients, $92(67.6 \%)$ and $44(32.4 \%)$ patients were classified into the initial-onset and early-onset LN groups, respectively. The initial-onset LN group had higher prevalences of impaired renal function $(34.8 \%$ vs. $11.4 \%, p=0.004)$ and microscopic hematuria $(73.9 \%$ vs. $54.5 \%, p=0.024)$ and higher urine protein/creatinine ratio (4626.1 [2180.0-6788.3] vs. 2410.0 $[1265.0-5168.5] \mathrm{mg} / \mathrm{g}, \mathrm{p}=0.006)$ at $\mathrm{LN}$ diagnosis. Renal relapse $(46.3 \%$ vs $25.7 \%, p=0.039$ ) and progression to chronic kidney disease (CKD) or end-stage renal disease (ESRD) were more common $(24.4 \%$ vs. $8.3 \%, p=0.042)$ in the initial-onset LN group. In the multivariable Cox regression analysis, initial-onset LN group had higher risk of renal relapse (adjusted hazard ratio [HR] 2.938, 95\% confidence interval [95\% $\mathrm{Cl}] 1.344-6.426, \mathrm{p}=0.007$ ) and progression to $\mathrm{CKD}$ or ESRD (adjusted HR 4.642, 95\% Cl 1.107-19.458, $p=0.036$ ), compared with early-onset LN group.

Conclusion: Patients with LN at SLE onset may have more severe renal presentations and worse renal outcome than those who develop $L N$ within 5 years.

References: Not applicable

Table. Hazard ratios for renal relapse and progression to CKD/ESRD according to onset time of $\mathrm{LN}$

\begin{tabular}{lccccc}
\hline & \multicolumn{2}{c}{ Univariable analysis } & & \multicolumn{2}{c}{ Multivariable analysis $^{\mathrm{a}}$} \\
\cline { 2 - 3 } & $\mathrm{HR}(95 \% \mathrm{Cl})$ & $\mathrm{p}$ & & $\mathrm{HR}(95 \% \mathrm{Cl})$ & $\mathrm{p}$ \\
\hline Renal relapse & & & & & \\
$\quad$ Early-onset LN & 1.000 (reference) & & & 1.000 (reference) & \\
Initial-onset LN & $2.734(1.315-5.686)$ & 0.007 & & $2.938(1.344-6.426)$ & 0.007 \\
Progression to CKD/ESRD & & & & & \\
$\quad$ Early-onset LN & 1.000 (reference) & & & 1.000 (reference) & \\
Initial-onset LN & $4.201(1.249-14.132)$ & 0.020 & & $4.642(1.107-19.458)$ & 0.036
\end{tabular}

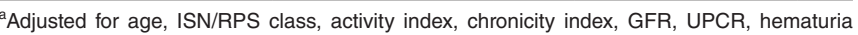
and use of $\mathrm{HCQ}$

LN, lupus nephritis; CKD, chronic kidney disease; ESRD, end-stage renal disease; ISN/RPS, International Society of Nephrology/Renal Pathology Society (ISN/RPS); GFR, glomerular filtration rate; UPCR, urine protein/creatinine ratio; HCQ, hydroxychloroquine; HR, hazard ratio; $\mathrm{Cl}$, confidence interval

Acknowledgments: None.

Disclosure of Interests: None declared

DOI: 10.1136/annrheumdis-2020-eular.4014

\section{THU0275 SEVERE PREECLAMPSIA RELATED TO ANTIPHOSPHOLIPID SYNDROME: AN EUROPEAN STUDY OF 40 WOMEN}

M. Larosa ${ }^{1}$, N. Morel ${ }^{2}$, M. Belhocine ${ }^{3}$, A. Ruffatti ${ }^{1}$, N. M. Silva ${ }^{4}$, R. Paul ${ }^{5}$ L. Mouthon ${ }^{2}$, M. Dreyfus ${ }^{6}$, J. C. Piette ${ }^{7}$, O. Souchaud-Debouverie ${ }^{8}$, C. DeneuxTharaux $^{9}$, V. Tsatsaris ${ }^{10}$, E. Pannier ${ }^{10}$, G. Guettrot Imbert ${ }^{2}$, V. Le Guern ${ }^{2}$, A. Doria', N. Costedoat-Chalumeau" ${ }^{2}{ }^{1}$ University of Padova, Rheumatology Unit; DIMED Department of Medicine, Padova, Italy; ${ }^{2}$ Cochin Hospital, Internal Medicine, Paris, France; ${ }^{3}$ Sacré-Coeur Hospital, Montréal, Canada; ${ }^{4}$ University Hospital Center of Caen, Internal Medicine Department, Caen, France; ${ }^{5}$ Foch Hospital, Internal Medicine, Paris, France; ${ }^{6}$ University Hospital Center of Caen, Gynecology and Obstetrics Department, Caen, France; ${ }^{7}$ Pitié-Salpêtrière Hospital, Paris, France; ${ }^{8}$ Poitiers University Hospital, Poitiers, France; ${ }^{9}$ INSERM U 1153, Paris, France $;{ }^{10}$ Cochin Hopital, Port-Royal Maternity, Paris, France
Background: One of the 3 features of obstetrical antiphospholipid syndrome (APS) is severe preeclampsia (PE). Its time of occurrence, the associated risk of thromboses and systemic lupus erythematosus (SLE) have not been reported yet.

Objectives: We analyzed severe PE in a series of women with APS.

Methods: We retrospectively collected data of female patients from 5 French internal medicine and 1 Italian rheumatology units. Inclusion criteria were: a severe PE/eclampsia(1), that occurred before 34 weeks of gestation (WG) in patients who met the APS classification criteria(2).

Results: 40 patients were enrolled (Table 1). Because of known APS/positive aPL/previous obstetrical complications, $23(57.5 \%)$ patients were treated during the index PE: 4 with low dose aspirin (LDA), 4 with low molecular weight heparin (LMWH), and 15 with a combination of both. 7 patients were also treated with hydroxychloroquine, 8 with corticosteroids and 3 with immunosuppressants. $17(42.5 \%)$ patients received no treatment. $24(60 \%)$ live births were observed. During a follow-up period of 3 years, 26(65\%) patients had at least 1 new pregnancy, with a total of 38 pregnancies which resulted in $33(86.8 \%)$ live births $57.5 \%$ pregnancies who resulted in live births occurred without any maternal or fetal complications. All 26 patients who had at least 1 pregnancy after index PE were treated with LDA; LMWH was given at prophylactic and therapeutic dosage in $13(50 \%)$ patients, respectively. No patient experienced 3 consecutive miscarriages.

\section{Table 1. 40 APS patients with severe PE}

\begin{tabular}{lc}
\hline Overall features (n, \%) & \\
Patients & $40(100)$ \\
Age at PE, (median, IQR) & $30.5(27-33)$ \\
PE term, WG (median, IQR) & $25.5(23-29)$ \\
Live births & $24(60)$ \\
Birth term, WG (median, IQR) & $25.5(23.7-30.3)$ \\
Associated SLE & $12(30)$ \\
Maternal complications (n, \%) & $25(62.5)$ \\
HELLP & $18(45)$ \\
E & $6(15)$ \\
CAPS & $3(7.5)$ \\
Placental abruptions & $3(7.5)$ \\
Fetal complications (n, \%) & $31(77.5)$ \\
IUGR & $18(45)$ \\
IUFD & $11(2.5)$ \\
Preterm delivery & $22(55)$ \\
Obstetrical history (n, \%) & \\
Primiparous & $21(52.5)$ \\
Index PE before APS & $12(30)$ \\
Thrombosis (n, \%) & \\
Thrombosis before PE index & $14(35.0)$ \\
Thrombosis after PE index & $2(5.0)$ \\
Abs at APS diagnosis (n, \%) & \\
aPL triple positivity & $21(52.5)$ \\
IgG/lgM anti-cardiolipin & $34(85.0)$ \\
IgG/IgM anti- $\beta 2 G P I$ & $25(62.5)$ \\
LAC & $33(82.5)$ \\
\hline
\end{tabular}

Legend to Table 1: PE: preeclampsia; APS: antiphospholipid syndrome; IQR: interquartil range; WG: weeks of gestation; SLE: systemic lupus erythematosus; HELLP: Hemolysis, elevated liver enzymes, low platelet; E: eclampsia; CAPS: catastrophic APS; IUGR: intrauterine growth restriction; IUFD: intrauterine fetal death; CHB: congenital atrioventricular block; aPL: antiphospholipid antibodies; LAC: lupus anticoagulant.

Conclusion: Among the APS criteria, "3 consecutive miscarriages criterion" was not found. The majority of patients also experienced thrombosis and SLE before the index PE.

References:

[1] Diagnosis and Management of preeclampsia and eclampsia. Internationa Journal of Gynecology \&Obestetrics 2002;77:67-75

[2] Miyakis S, et al. International consensus statement on an update of the classification criteria for definite antiphospholipid syndrome (APS). J Thromb Haemost 2006;4:295e 306.

Disclosure of Interests: Maddalena Larosa: None declared, Nathalie Morel: None declared, Meriem BELHOCINE: None declared, Amelia Ruffatti: None declared, Nicolas Martin Silva: None declared, Romain Paul: None declared, Luc Mouthon: None declared, Michel DREYFUS: None declared, Jean-Charles PIETTE: None declared, Odile Souchaud-Debouverie: None declared, Catherine Deneux-Tharaux: None declared, Vassilis Tsatsaris: None declared, Emmanuelle Pannier: None declared, Gaêlle Guettrot Imbert: None declared, Véronique LE GUERN Grant/research support from: UCB for GR2 study (to our institution), Andrea Doria Consultant of: GSK, Pfizer, Abbvie, Novartis, Ely Lilly, Speakers bureau: UCB pharma, GSK, Pfizer, Janssen, Abbvie, Novartis, Ely Lilly, BMS, Nathalie Costedoat-Chalumeau Grant/research support from: UCB to my institution

DOI: 10.1136/annrheumdis-2020-eular.5901 